\title{
Configurational forces in cyclic metal plasticity
}

\author{
Aris Tsakmakis ${ }^{1, *}$ and Michael Vormwald ${ }^{1, * *}$ \\ ${ }^{1}$ Materials Mechanics Group, Technische Universität Darmstadt, Franziska-Braun-Str. 3, D-64287 \\ Darmstadt, Germany
}

\begin{abstract}
The configurational force concept is known to describe adequately the crack driving force in linear fracture mechanics. It seems to represent the crack driving force also for the case of elastic-plastic material properties. The latter has been recognized on the basis of thermodynamical considerations. In metal plasticity, real materials exhibit hardening effects when sufficiently large loads are applied. Von Mises yield function with isotropic and kinematic hardening is a common assumption in many models. Kinematic and isotropic hardening turn out to be very important whenever cyclic loading histories are applied. This holds equally regardless of whether the induced deformations are homogeneous or non-homogeneous. The aim of the present paper is to discuss the effect of nonlinear isotropic and kinematic hardening on the response of the configurational forces and related parameters in elastic-plastic fracture problems.
\end{abstract}

\section{Introduction}

There are two possible approaches to introduce configurational forces and associated fundamental equations. The first one might be attributed to Morton E. Gurtin and coworkers (see e.g. [1] and the references cited there), while the second has been advocated especially by Gérard Maugin (see e.g. [2] and the references cited there). Interesting contributions have been provided among others by Herrmann and Kienzler [3], Müller et al. [4-6] and Steinmann [7]. Thermodynamical considerations in the framework of linear elastic fracture mechanics allow to interpret the configurational forces as crack driving forces and to establish an equivalent relation to the classical $J$-integral. It is worth mentioning, that, the use of the classical $J$-integral concept in elasto-plasticity restricted to special cases. Beside others, monotonic loading is assumed and the uniaxial stess-strain response has to be idealizied as non-linear elastic. The interesting property of configurational forces is, that they can be defined irrespective of the underlying material properties, implying their existence also for the case of elasto-plasticity. It has been argued in $[8,9]$ that a counterpart of the classical $J$-integral can be introduced in plasticity on the basis of the configurational force concept. This counterpart has been denoted as the incremental plasticity $J$-integral, $J^{\mathrm{ep}}$.

Fatigue crack growth in metal plasticity is induced by cyclic loading. For the prediction of cyclic loading phenomena it is known, that hardening effects in the material response

\footnotetext{
*e-mail: tsakmakis@wm.tu-darmstadt.de

**e-mail: vormwald@wm.tu-darmstadt.de
} 
are significant. Common plasticity models assume the existence of a von Mises yield function with isotropic and kinematic hardening. Thus it is of interest to discuss the influence of isotropic and kinematic hardening on the response of configurational forces and related fracture parameters. The paper aims to highlight such issues with reference to the Chaboche plasticity model.

\section{Chaboche plasticity model}

In the following, all tensorial components are referred to a Cartesian coordinate system $\left\{x_{\mathrm{i}}\right\}$ and the summation convention about repeated indices applies. Confining on small, isothermal deformations, plasticity models usually suppose the additive decomposition of the strain tensor $\varepsilon$ into elastic and plastic parts, $\varepsilon^{\mathrm{e}}$ and $\boldsymbol{\varepsilon}^{\mathrm{p}}$,

$$
\varepsilon_{\mathrm{ij}}=\varepsilon_{\mathrm{ij}}^{\mathrm{e}}+\varepsilon_{\mathrm{ij}}^{\mathrm{p}}
$$

to apply. Moreover, in metal plasticity the assumption is often made, that the elasticity law is independent of the previous plastic deformations, suggesting for the free energy per unit volume $\psi$ a decomposition of the form

$$
\psi=\psi^{\mathrm{e}}\left(\boldsymbol{\varepsilon}^{\mathrm{e}}\right)+\psi^{\mathrm{h}}(\boldsymbol{q})=\psi\left(\boldsymbol{\varepsilon}, \boldsymbol{\varepsilon}^{\mathrm{p}}, \boldsymbol{q}\right) .
$$

In this equation, $\psi^{\mathrm{e}}$ denotes the energy stored in the material due to elastic deformations, $\psi^{\mathrm{h}}$ is the energy stored in the material due to hardening effects and $\boldsymbol{q}$ denotes a set of scalar and tensorial hardening variables. Using standard thermodynamical methods the elasticity law

$$
\sigma_{\mathrm{ij}}=\frac{\partial \psi^{\mathrm{e}}}{\partial \varepsilon_{\mathrm{ij}}^{\mathrm{e}}}=\frac{\partial \psi}{\partial \varepsilon_{\mathrm{ij}}}
$$

can be established, where $\sigma$ is the Cauchy stress tensor. In the present paper linear isotropic elasticity is assumed by a quadratic potential $\psi^{\mathrm{e}}=\frac{1}{2} \varepsilon_{\mathrm{ij}}^{\mathrm{e}} \mathbb{C}_{\mathrm{ijk}} \varepsilon_{\mathrm{kl}}^{\mathrm{e}}$, with $\mathbb{C}$ being the isotropic fourth-order elasticity tensor, expressed in terms of Youngs's modulus $E$ and Poisson's ratio v. Thus, from Eq. (3),

$$
\sigma_{\mathrm{ij}}=\mathbb{C}_{\mathrm{ijkl}} \varepsilon_{\mathrm{kl}}^{\mathrm{e}} \text {. }
$$

Chaboche's plasticity model is adopted with a von Mises yield function with isotropic and kinematic hardening and with the associated normality condition for the plastic strain. Kinematic hardening is modeled by the Armstrong-Frederick law, while isotropic hardening is formulated by an exponential function of the plastic arc length. In the remainder of the paper, all

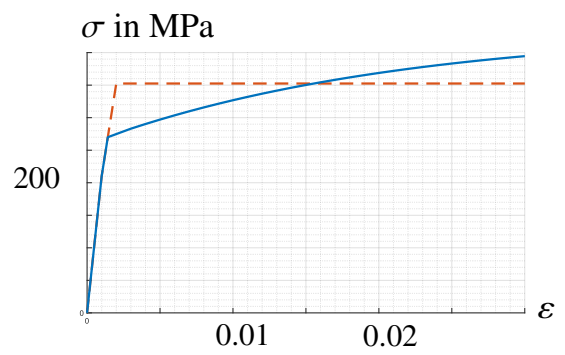

Figure 1. Pure isotropic and pure kinematic hardening (solid line) and ideal plasticity (dashed line). Coincident graphs according to pure isotropic and pure kinematic hardening. 

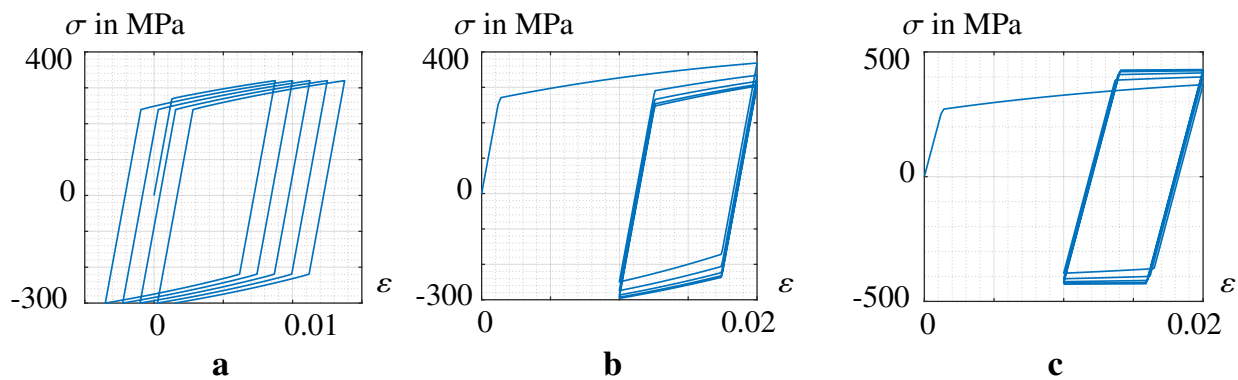

Figure 2. Uniaxial material responses. a Uniaxial ratchetting due to pure kinematic hardening. b Uniaxial cyclic relaxation due to pure kinematic hardening. c Uniaxial cyclic hardening due to pure isotropic hardening.

boundary value problems are calculated by the finite element program ABAQUS/CAE 2017. Only pure isotropic and pure kinematic hardening will be discussed, with material parameters chosen so, that the uniaxial stress-strain responses are coincident, see Fig. 1. The elasticity parameters are $E=210000 \mathrm{MPa}$ and $v=0.3$, the initial yield stress is $\sigma_{\mathrm{y}}=270 \mathrm{MPa}$ and the uniaxial tensile strength is $435 \mathrm{MPa}$. For reasons of comparison, ideal plasticity is also considered with yield stress $\sigma_{\mathrm{y}}=352,5 \mathrm{MPa}$, see Fig. 1 .

If uniaxial cyclic strain or stress controlled loading conditions apply, then the stress distributions will generally depend on the assumed hardening rule. For instance, it is well known for the assumed hardening laws, that the ratchetting phenomenon due to stress controlled loading with non-vanishing mean stress and the cyclic relaxation phenomenon due to strain controlled loading with non-vanishing mean strain can be predicted only by the kinematic hardening rule (see Figs. 2a,b), whereas the cyclic hardening phenomenon can be predicted only by the isotropic hardening rule (see Fig. 2c). Similar distributions apply also for corresponding plane strain loading conditions (see Figs. 3).

However, there are some distributions for uniaxial cyclic loading which are not reflected in a similar fashion in the corresponding plane strain case. As an example, consider uniaxial stress controlled loading with vanishing mean stress. According to isotropic hardening, only elastic responses arise for all cycles after the first unloading (see Fig. 4a), whereas stabilized hysteresis loop is predicted by pure kinematic hardening (see Fig. 4b). It can be recognized from Figs. $4 \mathrm{a}-4 \mathrm{~d}$, that the shape of the stress distributions in the plane strain case

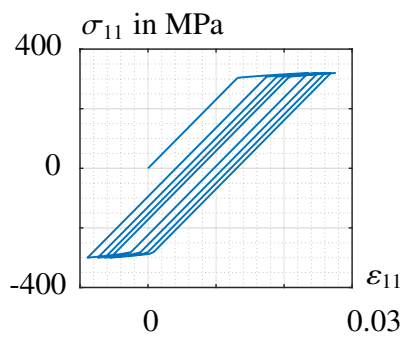

$\mathbf{a}$

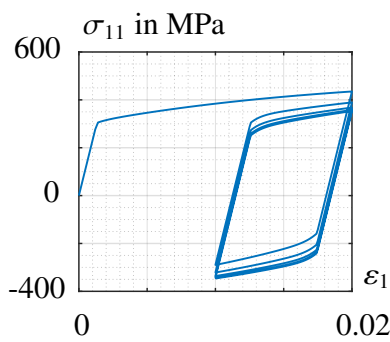

b

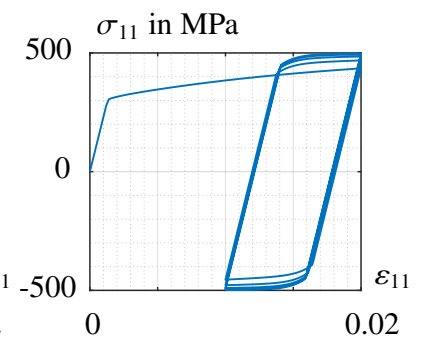

c

Figure 3. Plane strain material responses. a Plane strain ratchetting. b Plane strain cyclic relaxation. c Plane strain cyclic hardening 
is for pure kinematic hardening similar to that in the uniaxial case. However, this does not for pure isotropic hardening: Different to Fig. 4a, there are hysteresis loops present in Fig. 4c.

On the basis of the above discussions it is clear, that the hardening properties can significantly affect the predicted material responses. Therefore it is of interest to discuss whether the hardening properties affect parameters characterizing elastic-plastic fracture mechanics. Regarding configurational forces as driving forces, in some sense, for crack growth, attention will be focused on crack parameters expressed in terms of configurational forces. In view of the results for cyclic loading in this section, it is natural to expect some effect of the hardening proporties on crack parameters. One could also pose the reverse, perhaps academic question, which crack parameters reflect noticably different responses for pure isotropic and pure kinematic hardening, whenever cracks are subjected to cyclic loading. These issues will be discussed in the remainder of the paper.
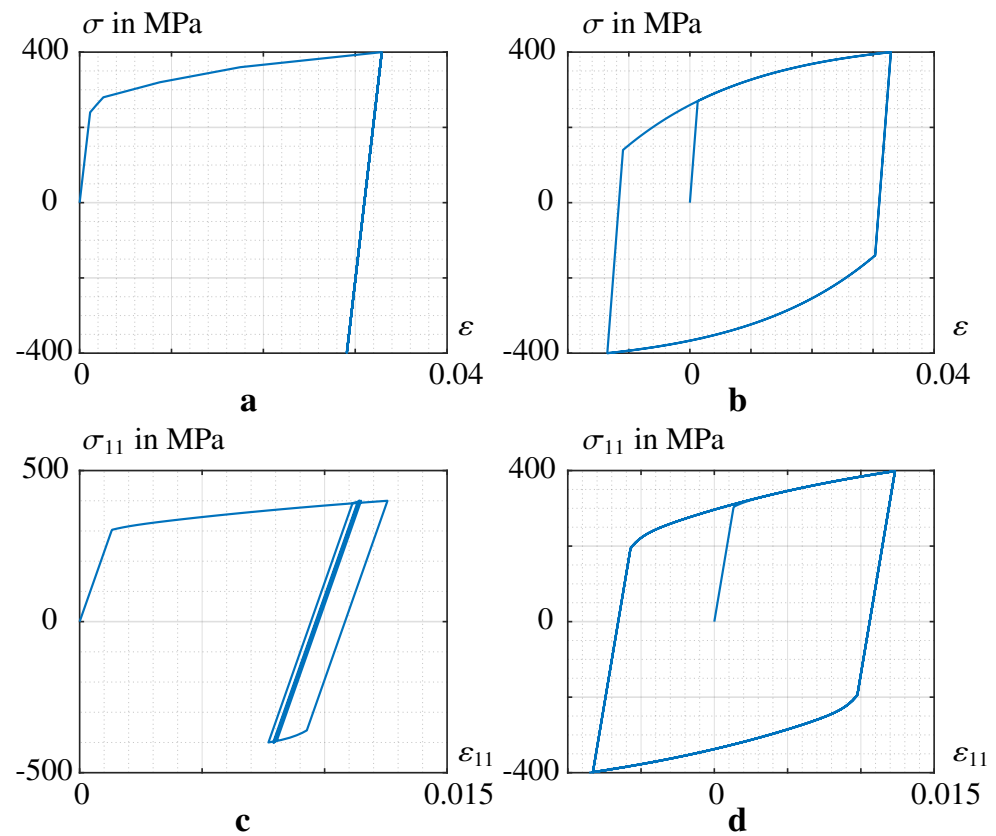

Figure 4. Stress distributions due to uniaxial cyclic loading with vanishing mean stress and corresponding counterparts for plane strain. a Pure isotropic hardening, uniaxial case. b Pure kinematic hardening, uniaxial case. c Pure isotropic hardening, plane strain case. d Pure kinematic hardening, plane strain case.

\section{Configurational forces}

Following [2] and [4] configurational forces can be introduced by considering the equilibrium equations with omitting body forces, $\sigma_{\mathrm{ij}, \mathrm{j}}=0$, where ()$_{\mathrm{j}, \mathrm{j}}$ denotes partial differentiation of () with respect to $x_{\mathrm{j}}$. Let $u_{\mathrm{i}}$ be the components of the displacement vector, so that $\varepsilon_{\mathrm{ij}}=\frac{1}{2}\left(u_{\mathrm{i}, \mathrm{j}}+u_{\mathrm{j}, \mathrm{i}}\right)$. After multiplication of the equilibrium equations with $u_{\mathrm{i}, \mathrm{k}}$, and rearrangement of terms, it follows that

$$
\left(\mathrm{u}_{\mathrm{i}, \mathrm{k}} \sigma_{\mathrm{ij}}\right)_{\mathrm{j}}-\varepsilon_{\mathrm{ij}, \mathrm{k}} \sigma_{\mathrm{ij}}=0 \Leftrightarrow\left(\mathrm{u}_{\mathrm{i}, \mathrm{k}} \sigma_{\mathrm{ij}}\right)_{\mathrm{j}}-\varepsilon_{\mathrm{ij}, \mathrm{k}} \frac{\partial \psi}{\partial \varepsilon_{\mathrm{ij}}}=0 .
$$


From Eq. (2), and since $\frac{\partial \psi^{\mathrm{e}}}{\partial \varepsilon_{\mathrm{ij}}^{\mathrm{e}}}=-\frac{\partial \psi^{\mathrm{e}}}{\partial \varepsilon_{\mathrm{ij}}^{\mathrm{p}}}$, it can be seen that

$$
\frac{\partial \psi}{\partial \varepsilon_{\mathrm{ij}}} \varepsilon_{\mathrm{ij}, \mathrm{k}}=\psi_{, \mathrm{k}}^{\mathrm{e}}-\frac{\partial \psi^{\mathrm{e}}}{\partial \varepsilon_{\mathrm{ij}}^{\mathrm{p}}} \varepsilon_{\mathrm{ij}, \mathrm{k}}^{\mathrm{p}} \Leftrightarrow \frac{\partial \psi}{\partial \varepsilon_{\mathrm{ij}}} \varepsilon_{\mathrm{ij}, \mathrm{k}}=\psi_{, \mathrm{k}}-\left(\psi_{, \mathrm{k}}^{\mathrm{h}}+\frac{\partial \psi^{\mathrm{e}}}{\partial \varepsilon_{\mathrm{ij}}^{\mathrm{p}}} \varepsilon_{\mathrm{ij}, \mathrm{k}}^{\mathrm{p}}\right) .
$$

There are two possibilities to proceed further (cf. [9]). The first one arises by incorporating the second of Eqs. (6) into the second of Eqs. (5),

$$
-\left(\psi \delta_{\mathrm{jk}}-\mathrm{u}_{\mathrm{i}, \mathrm{k}} \sigma_{\mathrm{ij}}\right)_{, \mathrm{j}}+\left(\psi_{, \mathrm{k}}^{\mathrm{h}}+\frac{\partial \psi^{\mathrm{e}}}{\partial \varepsilon_{\mathrm{ij}}^{\mathrm{p}}} \varepsilon_{\mathrm{ij}, \mathrm{k}}^{\mathrm{p}}\right)=0,
$$

while the second possibility arises by substituting the first of Eqs. (6) into the second of Eqs. (5),

$$
-\left(\psi^{\mathrm{e}} \delta_{\mathrm{jk}}-\mathrm{u}_{\mathrm{i}, \mathrm{k}} \sigma_{\mathrm{ij}}\right)_{, \mathrm{j}}+\frac{\partial \psi^{\mathrm{e}}}{\partial \varepsilon_{\mathrm{ij}}^{\mathrm{p}}} \varepsilon_{\mathrm{ij}, \mathrm{k}}^{\mathrm{p}}=0 .
$$

Here, $\delta_{\mathrm{ij}}$ denotes the Kronecker-Delta symbol. Eq. (7) suggests to interpret the term $\left(\psi \delta_{\mathrm{jk}}-\mathrm{u}_{\mathrm{i}, \mathrm{k}} \sigma_{\mathrm{ij}}\right)$ as configurational stresses associated with configurational volume forces $-\left(\psi_{, \mathrm{k}}^{\mathrm{h}}+\frac{\partial \psi^{\mathrm{e}}}{\partial \varepsilon_{\mathrm{ij}}^{\mathrm{p}}} \varepsilon_{\mathrm{ij}, \mathrm{k}}^{\mathrm{p}}\right)$ (cf. [9]), whereas, according to Eq. (8), ( $\left.\psi^{\mathrm{e}} \delta_{\mathrm{jk}}-\mathrm{u}_{\mathrm{i}, \mathrm{k}} \sigma_{\mathrm{ij}}\right)$ are configurational stresses associated with configurational volume forces

$$
\mathrm{f}_{\mathrm{k}}^{\mathrm{ep}}=-\frac{\partial \psi^{\mathrm{e}}}{\partial \varepsilon_{\mathrm{ij}}^{\mathrm{p}}} \varepsilon_{\mathrm{ij}, \mathrm{k}}^{\mathrm{p}},
$$

cf. $[9,10]$ and the references cited there. In the following the second possibility is discussed representatively. Assume a homogeneous body $\mathcal{B}$ in the plane strain setting to contain a crack and consider a path $\Gamma$ surrounding the crack tip, as indicated in $[8,10]$. Let $\mathcal{D}$ be the surface enclosed by $\Gamma$ and $\mathbf{e}$ the nominal crack growth direction. An elastic-plastic, path-depended $J$-integral, $J_{\Gamma}^{\text {ep }}$, may be introduced (cf. $[8,10]$ and the references cited there) through

$$
J_{\Gamma}^{\mathrm{ep}}=J_{\text {tip }}^{\mathrm{ep}}+\mathrm{e}_{\mathrm{k}} \int_{\mathcal{D} / \text { tip }} \mathrm{f}_{\mathrm{k}}^{\mathrm{ep}} \mathrm{dA},
$$

where

$$
J_{\text {tip }}^{\mathrm{ep}}=\mathrm{e}_{\mathrm{k}} \lim _{r \rightarrow 0} \int_{\Gamma_{\mathrm{r}}}\left(\psi^{\mathrm{e}} \delta_{\mathrm{jk}}-\mathrm{u}_{\mathrm{i}, \mathrm{k}} \sigma_{\mathrm{ij}}\right) \mathrm{m}_{\mathrm{j}} \mathrm{ds} .
$$

$\Gamma_{\mathrm{r}}$ is a contour drawn from the lower to the upper crank flank at a distance $\mathrm{r}$ from the crack tip and $\mathbf{m}$ is the outward unit normal vector on $\Gamma_{\mathrm{r}}$. It has been argued, that $J_{\Gamma}^{\mathrm{ep}}$ has the physical meaning of a true driving term in elastic-plastic materials. For small and large scale yielding conditions the contour $\Gamma$ has been identified by the crack tip plastic zone, PZ. The contour $\Gamma$ may influence significantly the magnitude of the corresponding $J$-integrals. Thus, one could suppose $\Gamma$ to be an active plastic zone, APZ, defined as the contour surrounding all material points in the neighborhood of the crack tip which actually experience plastic deformations. A further possibility is to define $\Gamma$ as an effective plastic zone, EPZ, defined as the contour surrounding all material points in the neighborhood of the crack tip which experience a given equivalent plastic strain after some fictitious crack growth. Such a definition for $J_{\mathrm{EPZ}}^{\mathrm{ep}}$ could eventually account for general yielding conditions as well. Because of the restricted length of the paper, we shall discuss in the next section exemplary only the effect of the hardening properties on $J_{\mathrm{PZ}}^{\mathrm{ep}}$ and $J_{\mathrm{APZ}}^{\mathrm{ep}}$. 


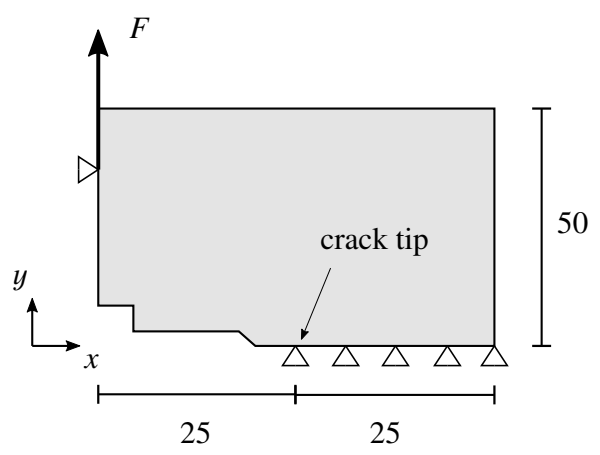

Figure 5. C(T)-specimen geometry. The rigid body is not depicted. All dimension are in mm.

\section{Effect of the hardening properties on $J_{\mathrm{PZ}}^{\mathrm{ep}}$ and $J_{\mathrm{APZ}}^{\mathrm{ep}}$}

The numerical determination of the configurational forces and $J^{\mathrm{ep}}$-integrals is done after solving the conventional boundary value problem with the finite element code ABAQUS. A selfwritten post-processing routine in the programing language Python is then used to calculate the distribution of the configurational forces in the body. The routine evaluates Eq. (8) with a numerical Gauss integration, following $[4,5]$. All simulations are conducted for the C(T)specimen depicted in Fig. 5 under plane strain conditions. Bilinear 4-node elements with a mesh size of $m=0.1 \mathrm{~mm}$ near the crack tip are used. Due to symmetry, only half of the specimen is modeled with a rigid body as a counterpart for possible crack flank contact. All

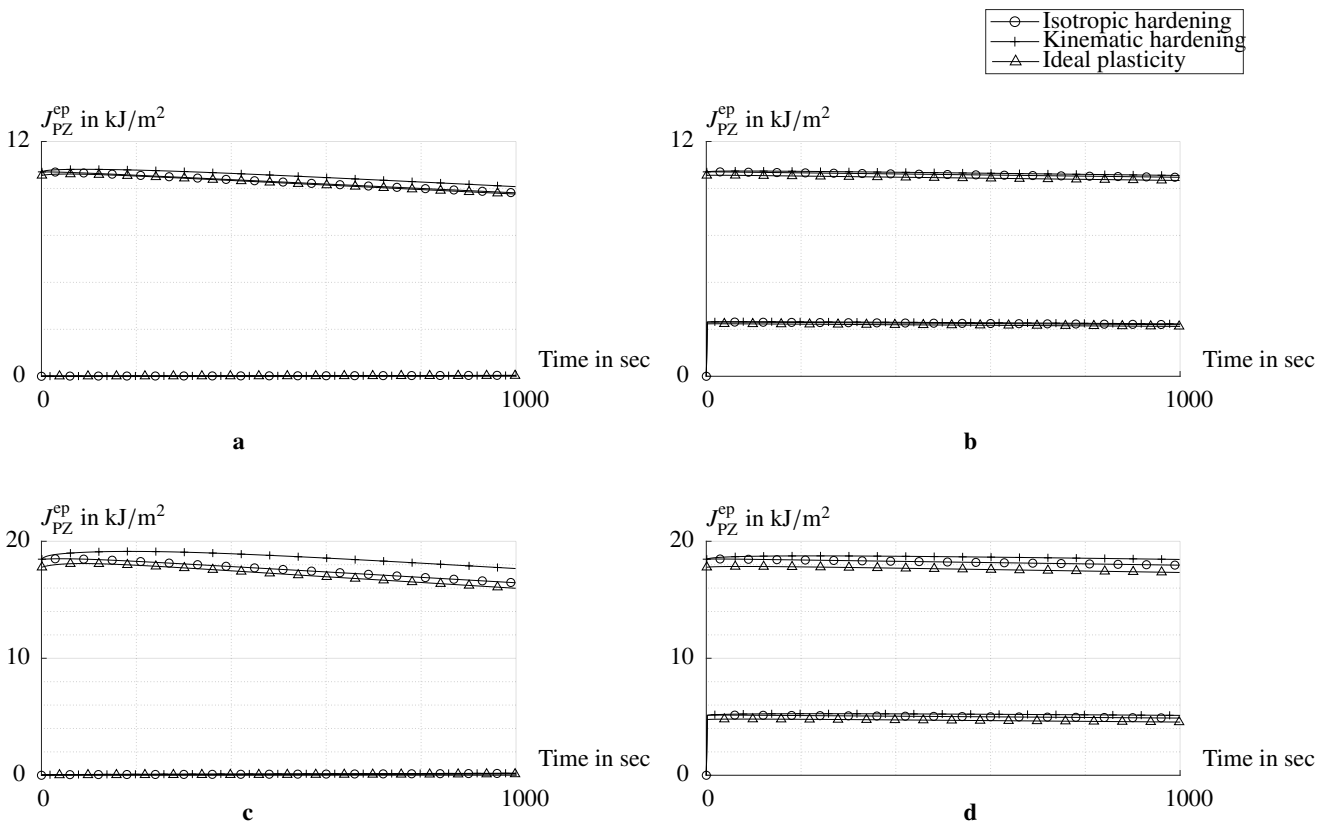

Figure 6. $J_{\mathrm{PZ}}^{\mathrm{ep}}$ values corresponding to $F_{\max }$ and $F_{\min }$ as function of time for 500 cycles. a $F_{\max }=27 \mathrm{kN}$, $R=0 . \mathbf{b} F_{\max }=27 \mathrm{kN}, R=0.5$. c $F_{\max }=35 \mathrm{kN}, R=0 . \mathbf{d} F_{\max }=35 \mathrm{kN}, R=0.5$. 

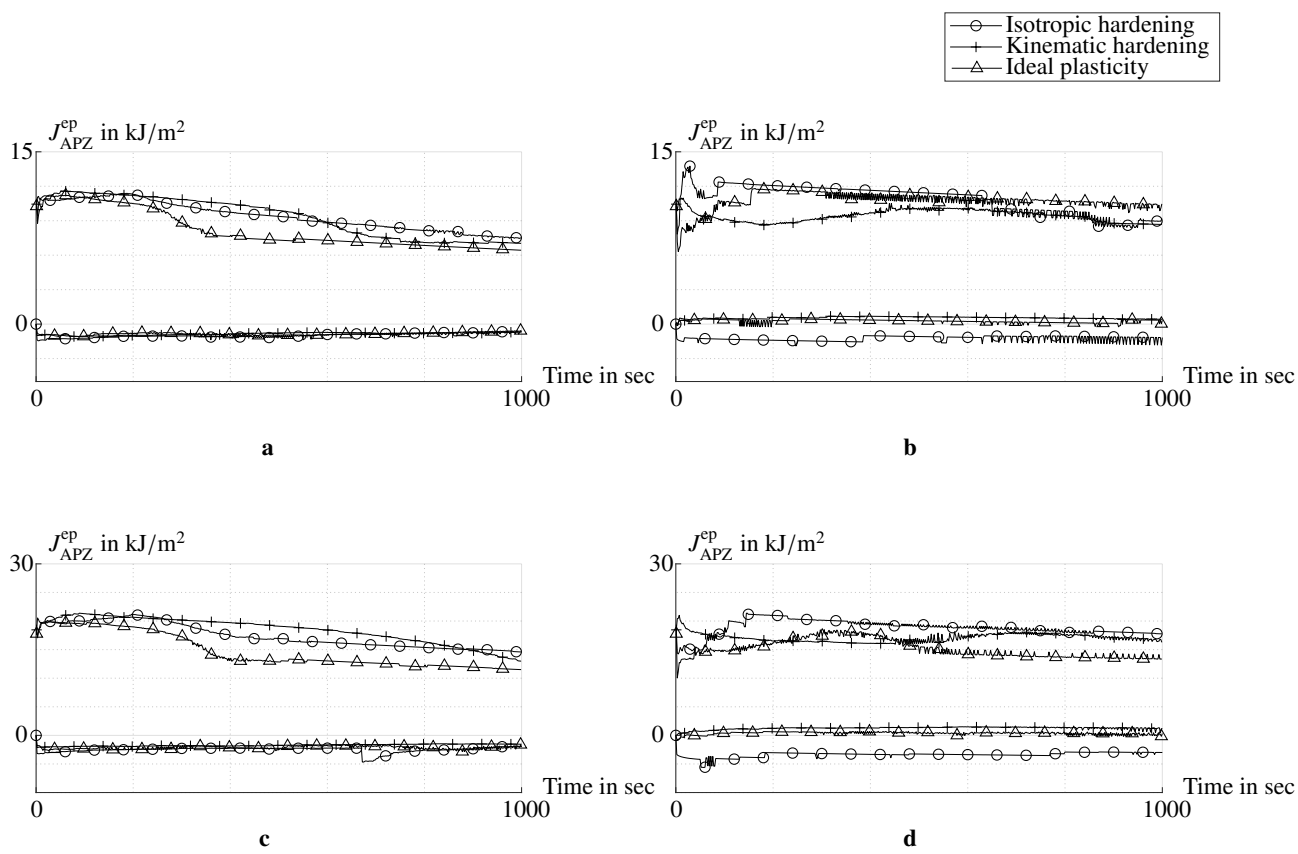

Figure 7. $J_{\mathrm{APZ}}^{\mathrm{ep}}$ values corresponding to $F_{\max }$ and $F_{\min }$ as function of time for 500 cycles. a $F_{\max }=27 \mathrm{kN}, R=0 . \quad$ b $F_{\max }=27 \mathrm{kN}, R=0.5 . \quad$ c $F_{\max }=35 \mathrm{kN}, R=0 . \quad$ d $F_{\max }=35 \mathrm{kN}$, $R=0.5$.

nodes on the ligament are fixed in $y$-direction and the load application point is fixed in $x$ direction. The material parameters are listed in section 2. Mode I, force controlled cyclic loading conditions are imposed with different magnitudes $\left(F_{\max }=27 \mathrm{kN}\right.$ and $\left.F_{\max }=35 \mathrm{kN}\right)$ and different $R$-ratios $R=F_{\min } / F_{\max }(R=0.5$ and $R=0)$.

Figs. 6a-d display predicted responses for $J_{\mathrm{PZ}}^{\mathrm{ep}}$ against time for 500 load cycles and different $F_{\max }$ and $R$ values. In all cases, large scale yielding conditions prevail. In each figure, the upper and lower graphs correspond to $F_{\max }$ and $F_{\min }$, respectively. The general tendency which can be stated, is that all graphs indicate qualitatively similar forms, and that the graphs according to ideal plasticity are below the ones according to isotropic hardening, which in turn are below the graphs according to kinematic hardening. The quantitative differences increase with increasing values of $F_{\max }$ and decrease with increasing values of $R$. Also, all $J_{\mathrm{PZ}}^{\mathrm{ep}}$ values for $F_{\max }=27 \mathrm{kN}$ are smaller than the corresponding values for $F_{\max }=35 \mathrm{kN}$. It is worth remarking, that all $J_{\mathrm{PZ}}^{\mathrm{ep}}$ values assigned to $F_{\min }$ are non-negative (cf. also [8]).

Figs. 7a-d show distributions for $J_{\mathrm{APZ}}^{\mathrm{e}}$ analogeous to those in Figs. 6a-d. Now, however, no regular tendencies in the forms of the graphs can be stated. It can be seen, that the graphs intersect each other and that negative $J_{\mathrm{APZ}}^{\mathrm{ep}}$ values appear. In comparison to Figs. 6a-d, it can be concluded, that the $J_{\mathrm{APZ}}^{\mathrm{ep}}$ values reflect bigger differences between the graphs attributed to the different hardening rules. In other words, $J_{\mathrm{APZ}}^{\mathrm{ep}}$ reflects more qualitative differences in the distributions induced by the three hardening models. The negative values for $J_{\mathrm{APZ}}^{\mathrm{ep}}$ at $F_{\text {min }}$ can be explained by the fact, that the integration contour is smaller than in case of Figs. 6, implying a stronger influence of the existing residual stresses. Generally, it should be noted, that some irregularities in the responses can be the consequence of the mesh discretization. To be more specific, as the integration contours are now smaller than in the case of $J_{\mathrm{PZ}}^{\mathrm{ep}}$ and the 
values of the stress and strain gradients in the vicinity of the crack tip are large, the accuracy of the numerical results will depend stronger on the mesh discretization.

\section{Concluding remark}

Configurational corces have turned out to represent an appropriate concept for defining parameters characterizing fracture mechanics. Some possibilities for related parameters have been discussed in the paper with reference to cyclic loading conditions. It has been shown, that some parameters reflect more sensitively differences arising for the asumed hardening hypothesis. A more detailed discussion, including further possibilities for configurational forces, will be given in a furthcoming paper.

\section{References}

[1] M.E. Gurtin, Archive for Rational Mechanics and Analysis 131, 67 (1995)

[2] G.A. Maugin, Configurational Forces: Thermomechanics, Physics, Mathematics, and Numerics (CRC Press, 2016)

[3] R. Kienzler, G. Herrmann, Mechanics in Material Space with Applications to Defect and Fracture Mechanics (Springer Science \& Business Media, 2012)

[4] R. Müller, K. Stefan, D. Gross, International Journal for Numerical Methods in Engineering 53, 1557 (2002)

[5] R. Müller, G. Dietmar, G.A. Maugin, Computational Mechanics 33, 421 (2004)

[6] R. Müller, G.A. Maugin, Computational mechanics 29, 52 (2002)

[7] R. Denzer, F.J. Barth, P. Steinmann, International Journal for Numerical Methods in Engineering 58, 1817 (2003)

[8] W. Ochensberger, Characterization of fatigue crack growth with the configurational force concept (2015), ISBN 978-3-18-334318-8

[9] N. Simha, F. Fischer, G. Shan, C. Chen, O. Kolednik, Journal of the Mechanics and Physics of Solids 56, 2876 (2008)

[10] O. Kolednik, R. Schöngrundner, F.D. Fischer, International Journal of Fracture 187, 77 (2014) 